CLINICAL STUDY

\title{
Evaluation of metabolic risk markers in polycystic ovary syndrome (PCOS). Adiponectin, ghrelin, leptin and body composition in hirsute PCOS patients and controls
}

\author{
Dorte Glintborg, Marianne Andersen, Claus Hagen, Jan Frystyk ${ }^{1}$, Veronica Hulstrøm² ${ }^{2}$, Allan Flyvbjerg ${ }^{1}$ \\ and Anne Pernille Hermann \\ Department of Endocrinology, Odense University Hospital, Klovervaenget 6, 3rd floor, 5000 Odense C, Denmark, ${ }^{1}$ Medical Research Laboratories, Clinical \\ Institute and Medical Department M (Diabetes and Endocrinology), Aarhus University Hospital, 8000 Aarhus C, Denmark and ${ }^{2}$ Department of Medical \\ Physiology, The Panum Institute, University of Copenhagen, 2200 Copenhagen N, Denmark
}

(Correspondence should be addressed to D Glintborg; Email: dorte.glintborg@dadlnet.dk)

\begin{abstract}
Objective: Polycystic ovary syndrome (PCOS) patients are abdominally obese and are at increased risk of developing the metabolic syndrome. Low adiponectin and ghrelin levels in PCOS patients could be caused by insulin resistance as well as high testosterone levels.

Design: Adiponectin and ghrelin levels were evaluated in 51 hirsute PCOS patients referred to the outpatient clinic of an academic, tertiary care medical centre and in 63 weight-matched female controls. Relationships between adiponectin, ghrelin, leptin, body composition, testosterone and insulin were examined.

Methods: Measurements of body composition including waist-hip-ratio (WHR), body mass index (BMI) and whole body dual-energy X-ray absorptiometry scan measures of body fat mass. Measurements of fasting levels of adiponectin, ghrelin, leptin, androgen status, oestradiol, lipid variables and insulin during follicular phase.

Results: Adiponectin levels were significantly decreased in obese PCOS patients compared with weightmatched controls (geometric mean ( -2 to 2 s.D.) $5.3(2.5-11.1)$ vs $7.3(3.0-17.4) \mathrm{mg} / \mathrm{l}, \mathrm{P}<0.05)$. Mean ghrelin was significantly lower in hirsute PCOS patients than in controls (0.6 (0.3 to 1.4) vs 0.8 (0.4 to 1.7$) \mu \mathrm{g} / \mathrm{l}, P<0.001)$ and this remained significant after subdividing subjects according to waist circumference and BMI. During multiple regression analysis, testosterone correlated positively with adiponectin and negatively with ghrelin independent of BMI, WHR and total fat mass.

Conclusion: Obese hirsute PCOS patients demonstrated significantly lower adiponectin levels than weight-matched controls suggesting a very high risk for the metabolic syndrome. Furthermore, ghrelin levels were decreased in hirsute PCOS patients and showed a significant, negative correlation with testosterone independent of body composition.
\end{abstract}

European Journal of Endocrinology $155337-345$

\section{Introduction}

An android fat distribution is observed not only in males but also in hyperandrogen females, most commonly those diagnosed with polycystic ovary syndrome (PCOS) (1). PCOS is a heterogeneous disease characterised by anovulation, hyperandrogenaemia and/or polycystic ovaries (2). Abdominal obesity is a feature of overweight PCOS patients (3) but is observed in most normal weight patients as well (4). Abdominal obesity is associated with insulin resistance, a high production of free fatty acids and an increased risk of type- 2 diabetes and cardiovascular disease (1). Previous studies found PCOS patients to have a five- to eight-fold increased risk for type-2 diabetes compared with healthy weightmatched females, thus making the syndrome of high socioeconomic importance $(5,6)$. Abdominal obesity is associated with changed secretion of several adipocytederived peptide hormones, commonly named adipocytokines. The production of adipocytokines has been shown to affect insulin sensitivity and to be an important predictor of the metabolic syndrome (7). Adiponectin is the most abundant adipocytokine and is mainly secreted from visceral fat cells (8). Adiponectin is the only protein expressed in adipose tissue that is downregulated in obesity (9). Studies showed that high adiponectin levels may protect against insulin resistance and type- 2 diabetes $(10,11)$ and adiponectin gene mutations were associated with increased type- 2 diabetes risk in Japanese subjects (12).

Ghrelin is a recently discovered peptide hormone with important effects on energy balance, food intake 
and weight regulation (13). Low ghrelin levels were found during conditions of positive energy balance such as obesity $(14,15)$ and accordingly, studies reported low ghrelin levels to be associated with insulin resistance and diabetes (16). Ghrelin was found to be expressed in pancreatic $\beta$ cells and may possibly be able to inhibit insulin secretion $(17,18)$.

Suggesting an increased risk of the metabolic syndrome in PCOS, previous studies have reported lower adiponectin and ghrelin levels in PCOS patients than in weight-matched controls $(8,11,18-23)$. Furthermore, significant correlations between androgen levels and adiponectin, and between androgen levels and ghrelin were found in some studies, thus further supporting the relationship between android characteristics in PCOS and metabolic risk factors.

However, PCOS is known to be a heterogeneous disease with a wide variety of clinical and paraclinical characteristics (2). When using the Rotterdam criteria, patients without clinical or biochemical hyperandrogenaemia may be diagnosed with PCOS (2). The inclusion of heterogeneous patient groups may explain why some studies found no significant associations between adiponectin, ghrelin and androgen levels in PCOS. Furthermore, to our knowledge, no previous studies have used more sophisticated methods to evaluate body composition nor evaluated these findings in relation to metabolic risk factors in PCOS.

In the present study, we evaluated the risk factors for the metabolic syndrome in PCOS. In order to describe relations between android characteristics and metabolic risk factors, only patients with clinical and paraclinical hyperandrogenaemia were included. The included patients were closely matched for body mass index (BMI) to a control group to examine further the metabolic characteristics in normal weight, overweight and obese subgroups of PCOS patients compared with controls. The importance of body composition for metabolic risk factors was evaluated by grouping individuals according to BMI and waist circumference and by including a whole body dual-energy X-ray absorptiometry (DXA) scan.

\section{Material}

During 2002-2003, 51 consecutive patients referred with a primary diagnosis of hirsutism to the outpatient clinic at the Department of Endocrinology, Odense University Hospital, were recruited. All patients were within the reproductive age, Caucasian and showed a Ferriman-Gallwey (FG) score above seven (24). Twentyseven patients had regular menses. Patients with a known medical disease including diabetes (fasting plasma glucose (p-glucose) $\geq 7.0 \mathrm{mmol} / \mathrm{l}$ ), hypertension, elevated liver enzymes or renal dysfunction were not included in the study. All patients had serum (s)prolactin and s-thyroid-stimulating hormone (s-TSH) within the reference intervals (RIs). S-17 hydroxyprogesterone levels were determined in all patients in order to exclude the adrenogenital syndrome. Two measurements of 24-h urine (U)-cortisol - or a short dexamethasone suppression test involving 2 days of decadron use $(1 \mathrm{mg})$ - were performed in patients with a clinical suspicion of Cushing's syndrome. A group of 63 healthy, Caucasian, reproductive-aged women were studied as controls. All the controls were thoroughly examined before inclusion in the study and all of them had regular menses and did not suffer from hyperandrogenaemia or hirsutism. The controls were recruited by advertisements at the local university, nursing school and at Odense University Hospital. The group of controls was matched to PCOS patients for BMI and age. The local ethics committee approved the study and all subjects gave written informed consent. Furthermore, the study complied with the Declaration of Helsinki. Data on bone mineral density and bone metabolic parameters in the study group have been published separately (25). All the patients and controls paused oral contraceptives for at least 6 months before evaluation and none were taking medicine known to affect hormonal or metabolic parameters. The patients and controls underwent a clinical examination including establishment of FG score, blood pressure, waist-hipratio (WHR), heart and lung stethoscopy, height and weight. All the participants were examined by the same doctor (Dorte Glintborg) and they lived in the County of Funen, Denmark. Fasting blood samples were drawn in the follicular phase (cycle days 2-8) in all controls and in patients with a cycle length shorter than 3 months; for patients with cycle length longer than 3 months, the blood samples were drawn on a random day. Blood samples were analysed for metabolic parameters (p-glucose, s-insulin, s-cholesterol, s-high density lipoprotein, s-triglyceride, s-low density lipoprotein), adipocytokines (leptin, adiponectin), ghrelin and hormone status (s-luteinizing hormone (s-LH), s-follicle-stimulating hormone (s-FSH), s-total (T)-testosterone, s-free (F)testosterone, s-sex-hormone-binding globulin (SHBG) and s-oestradiol). The homeostasis model of assessment (HOMA- $r=$ fasting blood glucose $\times$ fasting insulin/22.5) was calculated as an index of insulin resistance.

\section{DXA scan}

DXA in whole body array mode (DXA, Hologic QDR4500, Santax Medico, Aarhus C, Denmark) was used to measure whole body fat mass and to differentiate between extremital and central fat mass. Default software readings were used to divide the body into six compartments: head, trunk, arms (right and left) and legs (right and left). The trunk was defined by a horizontal line below the chin and vertical lines passing through colli femori. The arm regions were separated from the trunk at the levels of the shoulder joint. Body fat mass was determined for each region. Whole body 
fat mass was calculated by subtracting head fat mass from total fat mass. Technical performance was monitored by daily calibration scans using an anthropomorphic hologic phantom. The coefficient of variation (CV) for replicate scans of the same individual was $0.8 \%$ for whole body fat mass. The CV was determined by repeated scanning of 10 of the study participants.

\section{Assays}

Adiponectin was determined by an in-house time-resolved immunofluorometric assay as recently described (26). Total ghrelin was determined by an in-house RIA which yielding similar results as a commercially available assay from Phoenix Pharmaceuticals Inc. (St Joseph, MO, USA) (27). Leptin was determined using RIA kits purchased from Linco Research Inc. (St.Charles, Missouri, USA). The intra-assay CV was $7 \%$ for leptin. Serum levels of LH, FSH, insulin and oestradiol were analysed by time-resolved fluoroimmunoassays using commercial kits (AutoDELFIA, Perkin Elmer Life Sciences, Turku, Finland). Intra- and inter-assay variations are listed as ranges including established variations at both low and high concentrations. Intraassay variations were: FSH, 1.0-1.4\%; LH, 1.0-9.3\%; oestradiol $3.8-5.2 \%$ and insulin $2.1-3.7 \%$. Inter-assay variations were: FSH, $2.7-2.8 \%$; LH, $2.3-3.9 \%$; oestradiol, $3.7-8.5 \%$ and insulin, $3.4-4.0 \%$. Serum T- and F-testosterone, and SHBG were analysed using the specific RIA and extraction method as previously described (28). For this method, the intra-assay variation was $8.2 \%$ for T-testosterone and $5.2 \%$ for SHBG. The inter-assay variation was $13.8 \%$ for T-testosterone and $7.5 \%$ for SHBG. RIs in follicular phase were as follows: s-FSH 2.2-6.5 U/l, s-LH 1.6$9.3 \mathrm{U} / \mathrm{l}$, s-oestradiol $80-790 \mathrm{pmol} / \mathrm{l}$, s-T-testosterone $0.55-1.8 \mathrm{nmol} / \mathrm{l}$, s-F-testosterone $0.006-0.034 \mathrm{nmol} / \mathrm{l}$ and s-SHBG $41-170 \mathrm{nmol} / \mathrm{l}$. RIs for fasting insulin were $<55 \mathrm{pmol} / \mathrm{l}$, fasting plasma glucose $<7.0 \mathrm{mmol} /$ $\mathrm{l}$ and HOMA-r $<17.1 \mathrm{pmol} \mathrm{mmol} / \mathrm{l}^{-2}$.

\section{Calculations and statistical analysis}

Hormonal and body composition parameters were logGaussian distributed in healthy women and in patients. One-way ANOVA was used on log values to detect significant differences between several groups before the Bonferroni test was applied on subgroups. An unpaired $t$-test was used on $\log$ values to detect differences between two groups. Data were back-transformed and presented a geometric mean $(-2$ to +2 s.D. $)$ in tables as previously described by Altman and Bland (29).

Non-parametric tests were used for comparison of clinical characteristics between groups and data were expressed as median (25-75 quartiles). During univariate regression analysis, hormonal, body composition and metabolic parameters were log-transformed to achieve normally distributed parameters. Multiple regression analysis was performed using ghrelin and adipocytokines as the dependent variable. Patient group (patient: 1, control: 0) and hormonal/anthropometric parameters (T- and F-testosterone, insulin, adiponectin, ghrelin and leptin) were entered as independent variables in order to describe the correlations with adiponectin, ghrelin and leptin. These coefficients were then corrected for WHR, $\mathrm{BMI}$ or total fat mass, in order to differentiate hormonal effects independent of fat mass and body composition. Similar results were obtained when BMI and total fat mass were entered in the multiple regression analysis and only the results of total fat mass are included in the paper. Multiple regression analyses were also performed, including the interaction between peptide hormone (adiponectin, ghrelin or leptin) and patient group as an additional parameter (calculated as peptide hormone concentration $\times$ patient group). In these analyses, adiponectin, ghrelin or leptin was entered as the dependent variable and peptide hormone level, patient group and calculated interaction were entered as the independent variables. $P$-values $<0.05$ were considered to be significant. We used SPSS 13.0 (SPSS Inc, Chicago, USA) for calculations.

\section{Results}

\section{Adiponectin}

Obese, hirsute PCOS patients showed significantly lower adiponectin levels than obese controls, $P=0.04$ (Fig. 1). Adiponectin levels were significantly decreased in both patients and controls when BMI and waist circumference increased (Fig. 1, data for waist circumference not shown).

\section{Ghrelin}

The whole group of PCOS patients showed significantly lower ghrelin concentrations than controls, which remained significant when comparing overweight and obese patients with weight-matched controls. When subdividing patients according to waist circumference, both subgroups of patients showed significantly lower ghrelin levels than matched controls (for waist $\leq 88 \mathrm{~cm}$, patients showed ghrelin $0.7 \quad(0.3-1.8)$ vs $1.0 \quad(0.5-1.9) \quad \mu \mathrm{g} / \mathrm{l}$ in controls, $P=0.004$. For waist $>88 \mathrm{~cm}$, ghrelin levels were $0.5(0.3-1.0)$ in patients vs $0.7(0.4-1.4) \mu \mathrm{g} / \mathrm{l}$ in controls, $P=0.001)$. Ghrelin levels were significantly decreased in both patients and controls when BMI and waist circumference increased (Fig. 1).

\section{Leptin}

Leptin levels increased significantly when BMI increased in patients and controls (Fig. 1). 

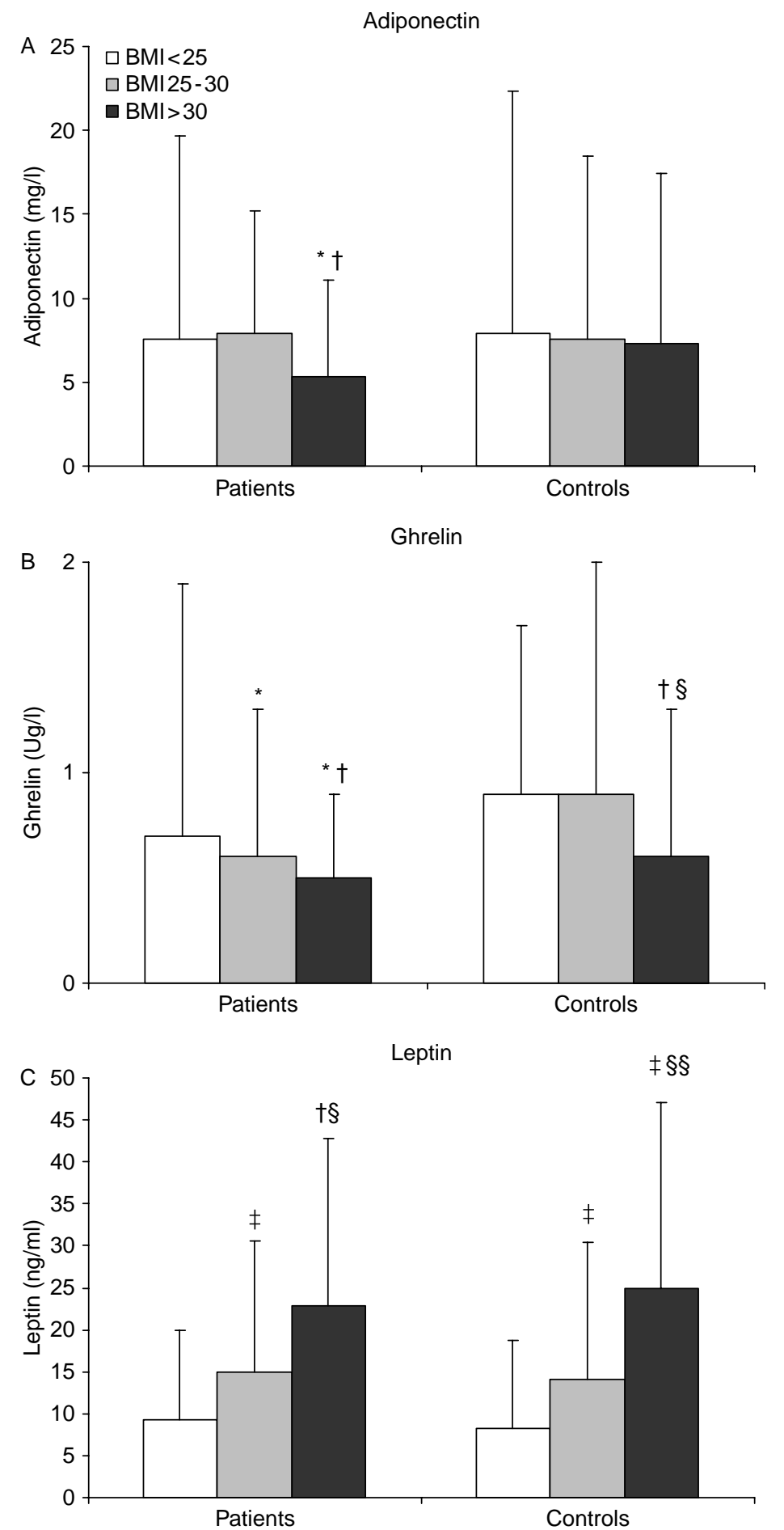

Figure 1 Peptide hormone levels in patients and controls divided according to BMI. Data presented as geometric mean ( +2 s.D.) ${ }^{*} P<0.05$ vs controls in same weight group. ${ }^{\dagger} P<0.001{ }^{\ddagger} P<0.05$ vs subjects with $\mathrm{BMl}<25$ within patient/control group. ${ }^{\S \S} P<0.001{ }^{\S} P<0.05$ vs subjects with BMI 25-30 within patient/control group.

\section{Insulin and HOMA-r}

Total FG score among PCOS patients was 11 (9-14), median (25-75 quartile). Patients with normal weight and patients with waist circumference $\leq 88 \mathrm{~cm}$ had significantly higher HOMA-r values compared with controls (for normal weight individuals, HOMA-r values were $8.0(4.0-16.0)$ vs $5.3(2.1-13.6) \mathrm{pmol}$ $\mathrm{mmol} / \mathrm{l}^{-2}, P=0.002$ and for waist $\leq 88 \mathrm{~cm}$, HOMA- $r$ were $8.1(3.6-18.4)$ vs $5.6(2.1-14.5), P=0.004)$ (Table 1). 
Table 1 Body composition, hormonal and metabolic characteristics in patients and controls. Data presented as geometric mean ( -2 to +2 S.D.).

\begin{tabular}{|c|c|c|}
\hline & $\begin{array}{l}\text { Hirsute PCOS } \\
\text { patients }(n=51)\end{array}$ & Controls $(n=63)$ \\
\hline Ghrelin $(\mu \mathrm{g} / \mathrm{l})$ & $0.6(0.3-1.4)^{\star \star}$ & $0.8(0.4-1.7)$ \\
\hline Adiponectin (mg/l) & $6.9(2.9-16.4)$ & $7.6(3.0-19.4)$ \\
\hline Leptin (ng/ml) & $14.2(5.2-39.2)$ & $12.8(4.1-40.3)$ \\
\hline $\begin{array}{l}\text { Total testosterone } \\
(\mathrm{nmol} / \mathrm{l})\end{array}$ & $1.59(0.72-3.51)^{\star}$ & $1.27(0.67-2.40)$ \\
\hline $\begin{array}{l}\text { Free testosterone } \\
\qquad(\mathrm{nmol} / \mathrm{l})\end{array}$ & $0.031(0.011-0.086)^{\star \star}$ & $0.021(0.017-0.026)$ \\
\hline SHBG (nmol/l) & $39(13-116)^{\star \star}$ & $56(24-129)$ \\
\hline Glucose (mmol/l) & $5.1(4.1-6.4)$ & $5.2(4.1-6.6)$ \\
\hline Insulin (pmol/l) & $46(17-126)^{\star}$ & $34(11-105)$ \\
\hline $\begin{array}{l}\mathrm{HOMA}-r(\mathrm{pmol} \\
\mathrm{mmol} / \mathrm{l}-2)\end{array}$ & $10.3(3.4-30.9)^{\star}$ & $7.9(2.3-27.6)$ \\
\hline \multicolumn{3}{|l|}{ Body composition } \\
\hline BMI $\left(\mathrm{kg} / \mathrm{m}^{2}\right)$ & $27.1(17.8-41.8)$ & $27.5(19.1-39.6)$ \\
\hline WHR & $0.81(0.67-0.98)^{\star}$ & $0.78(0.67-0.93)$ \\
\hline Waist (cm) & $87(66-115)$ & $86(63-118)$ \\
\hline Total fat mass $(\mathrm{kg})$ & $23.6(11.1-50.3)$ & $23.4(10.4-55.2)$ \\
\hline $\begin{array}{l}\text { Central fat mass } \\
(\mathrm{kg})\end{array}$ & $11.1(4.6-27.0)$ & $10.8(4.0-29.1)$ \\
\hline $\begin{array}{l}\text { Extremital fat } \\
\text { mass } \\
\quad(\mathrm{kg})\end{array}$ & $12.3(6.2-24.7)$ & $12.9(6.1-27.3)$ \\
\hline
\end{tabular}

${ }^{\star} P<0.05$ vs controls, ${ }^{\star \star} P<0.001$ vs controls.

\section{Bivariate regression analysis}

Adiponectin In hirsute PCOS patients, adiponectin levels correlated negatively with BMI, WHR, DXA-scan established measures of fat mass, insulin and HOMA-r levels. Adiponectin correlated positively with ghrelin and negatively with leptin. In controls, adiponectin correlated inversely with WHR and positively with T-testosterone (Table 2).
Ghrelin Ghrelin levels showed significant, negative correlations with BMI, DXA-scan established measures of fat mass, insulin, F-testosterone and HOMA-r in PCOS patients and controls. In PCOS patients, a significant positive correlation was found between adiponectin and ghrelin, whereas ghrelin correlated negatively with leptin. In controls, only the correlation between ghrelin and leptin was significant.

Leptin In both PCOS patients and controls, leptin levels correlated significantly with measures of body fat, fat distribution, insulin and HOMA-r.

F-testosterone In PCOS patients, F-testosterone levels showed a significant, positive correlation with waist circumference $(r=0.279, P<0.05)$ and with central fat mass $(r=0.305, P<0.05)$.

\section{Multiple regression analysis}

During multiple regression analyses, adiponectin, ghrelin and leptin were entered as the dependent variables as described in the Statistical analysis section (Table 3).

Adiponectin A significant correlation was found between adiponectin and T-testosterone after correcting for patient group, which remained significant after adjusting for WHR or total fat mass. A significantly negative correlation was found between adiponectin and insulin, which remained significant after adjusting for WHR or fat mass. A significant interaction was found between ghrelin $\times$ patient group and adiponectin $(B=0.336, P=0.03)$.

Table 2 Pearson correlation tests of body composition parameters and sex hormones to ghrelin, adiponectin and leptin in patients and controls.

\begin{tabular}{|c|c|c|c|c|c|c|}
\hline & \multicolumn{3}{|c|}{ Hirsute PCOS patients } & \multicolumn{3}{|c|}{ Controls } \\
\hline & Ghrelin & Adiponectin & Leptin & Ghrelin & Adiponectin & Leptin \\
\hline Total testosterone & -0.09 & 0.17 & 0.03 & -0.24 & $0.25^{\star}$ & -0.13 \\
\hline Free testosterone & $-0.32^{\star}$ & -0.05 & 0.23 & $-0.41^{*}$ & -0.09 & 0.06 \\
\hline SHBG & $0.42^{\star *}$ & $0.40^{\star *}$ & $-0.36^{\star \star}$ & $0.27^{\star}$ & $0.39^{* *}$ & -0.24 \\
\hline Insulin & $-0.51^{\star \star}$ & $-0.62^{* *}$ & $0.55^{\star *}$ & $-0.28^{*}$ & -0.24 & $0.72^{* *}$ \\
\hline HOMA-r & $-0.48^{\star \star}$ & $-0.59^{\star \star}$ & $0.58^{\star \star}$ & $-0.29^{\star}$ & -0.24 & $0.73^{\star \star}$ \\
\hline BMI & $-0.49^{\star \star}$ & $-0.36^{\star \star}$ & $0.82^{* *}$ & $-0.35^{\star}$ & -0.16 & $0.82^{\star \star *}$ \\
\hline WHR & -0.27 & $-0.42^{\star \star}$ & $0.35^{\star *}$ & $-0.29^{\star}$ & $-0.28^{*}$ & $0.51^{\text {** }}$ \\
\hline Waist & -0.44 & $-0.46^{\star *}$ & $0.75^{\star \star}$ & $-0.35^{\star *}$ & -0.20 & $0.81^{* *}$ \\
\hline Total fat mass & $-0.52^{\star *}$ & $-0.38^{* *}$ & $0.80^{\star *}$ & $-0.34^{\star *}$ & -0.13 & $0.86^{\star *}$ \\
\hline Central fat mass & $-0.57^{\star *}$ & $-0.46^{\star *}$ & $0.85^{\star *}$ & $-0.31^{*}$ & -0.23 & $0.87^{\star \star}$ \\
\hline Extr. fat mass & $-0.42^{\star \star}$ & -0.26 & $0.85^{\star \star}$ & $-0.35^{\star \star}$ & -0.02 & $0.81^{\star \star}$ \\
\hline Ghrelin & - & $0.56^{\star *}$ & $-0.48^{\star *}$ & - & -0.001 & $-0.26^{*}$ \\
\hline Adiponectin & $0.56^{\star \star}$ & - & $-0.37^{\star \star}$ & -0.001 & - & -0.08 \\
\hline Leptin & $-0.48^{\star *}$ & $-0.37^{*}$ & - & $-0.26^{*}$ & -0.08 & - \\
\hline
\end{tabular}

SHBG, sex-hormone-binding globulin; HOMA-r, homeostatic model of assessment of insulin resistance; BMI, body mass index; WHR, waist-hip-ratio.

${ }^{\star} P<0.05,{ }^{* \star} P<0.01$. All variables are log transformed. 
Table 3 Multiple regression analysis with adiponectin, ghrelin and leptin as the dependent variable. Entered independent variables: total and free testosterone, insulin, adiponectin, ghrelin, leptin. Only data from models with significant $R^{2}$ values are presented. Data presented as $B$-coefficient $\left(P\right.$-level) for the hormone in the left column entered as the independent variable. The $R^{2}(P$-level) for the whole regression model is presented in the right columns.

\begin{tabular}{|c|c|c|c|c|c|c|}
\hline & \multirow[b]{2}{*}{$B$-coefficient ${ }^{a}$} & \multirow[b]{2}{*}{$R^{2}$} & \multicolumn{2}{|c|}{ WHR adjusted } & \multicolumn{2}{|c|}{ Total fat mass adjusted } \\
\hline & & & $B$-coefficient ${ }^{\mathrm{b}}$ & $R^{2}$ & $B$-coefficient ${ }^{c}$ & $R^{2}$ \\
\hline \multicolumn{7}{|l|}{ Log (ghrelin) } \\
\hline F-testosterone & $-2.20^{\star}$ & $0.19^{\star *}$ & $-1.95^{\star}$ & $0.24^{\star \star}$ & $-1.76^{\star}$ & $0.31^{\star \star}$ \\
\hline Insulin & $-0.002^{\star *}$ & $0.28^{\star *}$ & $-0.002^{\star}$ & $0.28^{\star *}$ & $-1.410-3$ & $0.31^{* *}$ \\
\hline Leptin & $-7.4 \times 10^{-3 \star *}$ & $0.26^{\star *}$ & $-6.1 \times 10^{-3 * *}$ & $0.27^{\star *}$ & $-1.1 \times 10^{-3}$ & $0.29^{\star \star}$ \\
\hline Adiponectin & $1.3 \times 10^{-2 \star *}$ & $0.22^{* *}$ & $9.4 \times 10^{-3 \star *}$ & $0.24^{\star \star}$ & $8.7 \times 10^{-3 \star \star}$ & $0.31^{\star *}$ \\
\hline \multicolumn{7}{|l|}{ Log (adiponectin) } \\
\hline T-testosterone & $0.075^{\star}$ & $0.053^{*}$ & $0.065^{*}$ & $0.13^{\star \star}$ & $0.072^{\star \star}$ & $0.10^{*}$ \\
\hline Insulin & $-0.003^{\star *}$ & $0.16^{\star \star}$ & $-0.002^{\star}$ & $0.19^{\star *}$ & $-3.4 \times 10^{-3 * *}$ & $0.17^{\star *}$ \\
\hline Ghrelin & $0.29^{\star}$ & $0.077^{\star}$ & $7.9 \times 10^{-2}$ & $0.14^{\star \star}$ & $9.5 \times 10^{-2}$ & $0.09^{*}$ \\
\hline \multicolumn{7}{|l|}{ Log (leptin) } \\
\hline Insulin & $0.005^{\star *}$ & $0.36^{\star *}$ & $0.005^{\star *}$ & $0.38^{\star *}$ & $7.5 \times 10^{-4}$ & $0.69^{\star *}$ \\
\hline Ghrelin & $-0.22^{\star \star}$ & $0.10^{\star}$ & $-0.15^{\star}$ & $0.23^{\star \star}$ & $2.6 \times 10^{-3}$ & $0.71^{\star \star *}$ \\
\hline
\end{tabular}

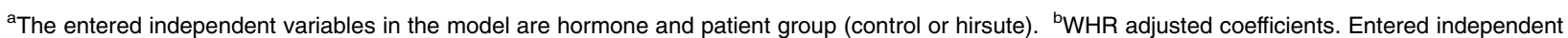
variables: WHR, hormone and patient group (control or hirsute). ${ }^{\mathrm{C}}$ Fat mass adjusted coefficients. Entered independent variables: Fat mass, hormone and patient group (control or hirsute). ${ }^{\star} P<0.05,{ }^{\star \star} P<0.001$.

Ghrelin F-testosterone, insulin and leptin showed significantly negative correlations with ghrelin after correcting for patient group in the regression analyses, whereas a positive correlation was found when entering adiponectin. These correlations remained significant after correcting for WHR. When the regression model was corrected for total fat mass, only the correlations with F-testosterone and adiponectin remained significant. When ghrelin was included as the dependent variable and the interaction between leptin or adiponectin and patient group was entered as the independent variable as described in the Statistical analysis section, a significant interaction was found between adiponectin $\times$ patient group and ghrelin $(B=0.003$, $P=0.01)$.

Leptin Insulin and ghrelin showed significant correlations with leptin after correcting for patient group and WHR, whereas no significant correlations remained after correcting for fat mass.
The correlations between adiponectin, ghrelin, leptin, testosterone and insulin resistance in PCOS patients are summarised in Figure 2.

\section{Discussion}

In the present study, obese hirsute PCOS patients showed modest but significantly lower adiponectin levels than weight-matched obese controls, and adiponectin levels significantly decreased as BMI and WHR increased. These results suggest a very high risk for the metabolic syndrome in obese PCOS patients and support that metabolic screening is of high relevance especially in this subgroup of patients. Our findings of significantly lower adiponectin levels in PCOS than in weightmatched controls as well as the close, inverse relation between adiponectin and BMI have previously been reported $(8,10,11,23,30-32)$.

In the present study, however, we further evaluated the importance of body composition for metabolic risk factors and performed whole body DXA scan measures

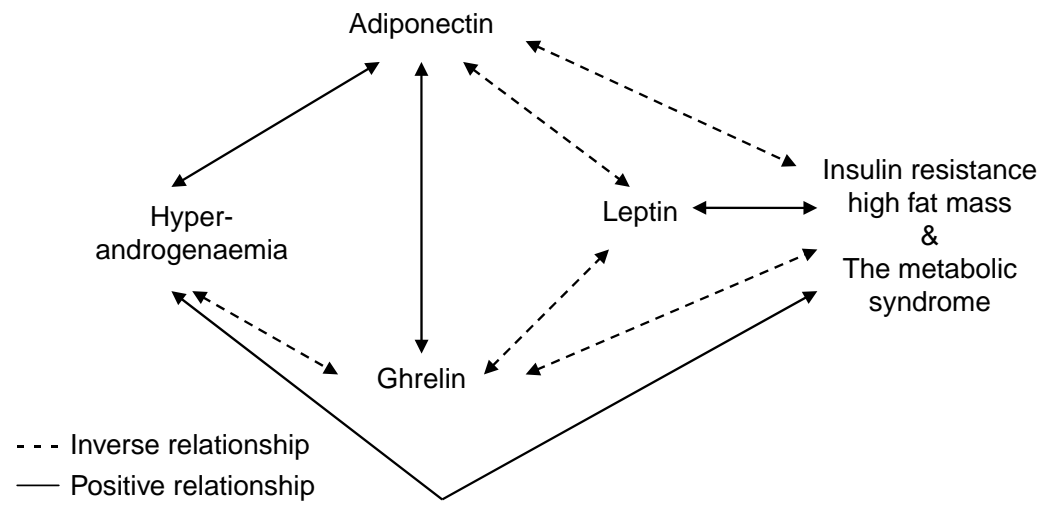

Figure 2 Summary of relations between adiponectin, ghrelin, leptin and testosterone, insulin resistance and fat mass in hirsute PCOS patients. 
in all the included individuals. In PCOS patients, the inverse correlation between adiponectin and central fat mass was even stronger than the correlation observed between adiponectin and BMI, thus further supporting the importance of abdominal fat mass for the secretion of adiponectin (33). To our knowledge, we are the first to apply DXA scan established measures of fat distribution and to correlate these measures with adiponectin levels in PCOS.

Fasting insulin levels were inversely correlated with adiponectin and this association remained significant after adjusting for BMI $(10,11)$. Notably, adiponectin levels were negatively correlated with measures of fat mass, insulin and HOMA-r levels in PCOS patients, whereas in controls only the correlations between adiponectin and WHR remained significant. These results are in agreement with a study by Sepilian et al. (22) describing significantly inverse associations between adiponectin and measures of insulin resistance in PCOS, whereas no significant correlations were found in controls.

A significant, positive correlation between testosterone and adiponectin was found only in controls. However, during multiple regression analysis a fat mass-independent positive association was found between testosterone and adiponectin in patients as well as controls. These results may seem conflicting as previous studies have described significantly higher adiponectin levels in women than in men $(34,35)$ and furthermore, that testosterone supplementation was followed by decreased adiponectin levels (34, 36). However, our data are supported by two previous studies finding significant positive associations between adiponectin and testosterone levels in $\operatorname{PCOS}(11,32)$. Vribkova et al. suggested that this association was caused by a more complex interrelation between testosterone, oestradiol and adiponectin in PCOS (32). In the present study, we found significantly decreased oestradiol levels in our patient population, which may have affected results (25). The patients in the present study were characterised by insulin resistance as well as hyperandrogenaemia. The fact that, in the present study, adiponectin levels tended to be lower in hyperandrogen patients than in controls suggests that the positive relation between adiponectin and testosterone was weaker than the inverse relation between adiponectin and insulin. The present data may suggest that high testosterone levels in our patient population prevented even lower adiponectin levels and may help explain why significantly decreased adiponectin levels were not found in all subgroups of patients compared with controls. Furthermore, only modest differences were found between adiponectin levels in patients and controls. Type 1 error may be an alternate explanation why significantly impaired adiponectin levels were observed only in obese PCOS patients. Future studies are needed to clarify the relationships between adiponectin, testosterone and insulin in PCOS.
We found moderately, but significantly decreased serum ghrelin levels in all subgroups of PCOS patients compared with controls and ghrelin levels showed a significant, negative correlation with F-testosterone levels independent of body composition measures.

Ghrelin was first discovered in 1999 (13) and is mainly secreted from the entero-endocrine cells of the stomach (37). Low ghrelin levels were associated with increased insulin levels and increased diabetes risk, thus suggesting ghrelin to be an independent risk factor of type-2 diabetes (16).

Supporting the hypothesis of a negative association between ghrelin and risk factors of the metabolic syndrome, we found a significantly inverse correlation between measures of insulin resistance and ghrelin in PCOS patients and controls. Previous studies found decreased ghrelin levels in insulin resistant PCOS patients compared with weight-matched controls (18-21), but in these studies, BMI and WHR were the only available measures of body composition. The inclusion of more exact body composition measurements is therefore needed to characterise further the relationships between ghrelin, fat mass and the metabolic syndrome. When we added the results of the DXA scan-established measures of body composition, ghrelin showed significantly negative correlations with total, central and extremital fat mass in PCOS patients and controls, with the highest correlation coefficient found for central fat mass in patients. No significant associations between insulin or HOMA-r and ghrelin remained after adjusting for fat mass, thus suggesting fat mass to be of higher importance for ghrelin secretion than insulin. Orio et al. found higher fasting insulin levels in PCOS patients compared with controls but similar ghrelin levels (38), thus supporting a minor importance of insulin for ghrelin secretion. Orio et al. and Moran et al. demonstrated significantly inverse correlations between ghrelin and BMI in PCOS, whereas correlations between insulin and ghrelin were nonsignificant $(19,38)$. Following a hypocaloric diet combined with metformin treatment, Pagotto et al. found no significant changes in ghrelin levels despite significantly improved insulin sensitivity (21).

Using an accurate testosterone assay, we found a significantly inverse association between F-testosterone and ghrelin, which remained significant after adjusting for WHR or total fat mass. To our knowledge, we are the first to demonstrate a fat-independent relationship between testosterone and ghrelin levels in PCOS, but few previous studies have demonstrated significant bivariate correlations between ghrelin and testosterone in PCOS patients $(21,39)$. In most studies, no significant correlations were found between ghrelin and testosterone in $\operatorname{PCOS}(18,20,32,38)$. The inclusion of heterogeneous patient groups including hyperandrogen as well as non-hyperandrogen PCOS patients and the use of commercially available RIA kits for the evaluation of testosterone levels may have 
affected results in some of these studies. The ghrelin receptor is distributed not only in the central nervous system but also in ovarian tissue, thus suggesting a possible reproductive function for ghrelin (40). The association between ghrelin and reproductive function was supported by a recent placebo-controlled study reporting significantly increased ghrelin levels following decreased testosterone levels during flutamide therapy in PCOS patients (41).

We found significantly higher leptin levels when BMI and waist circumference increased in both patients and controls and in accordance with previous studies, close relations were demonstrated between leptin and measures of fat mass and insulin sensitivity $(8,42-$ 47). Our findings of insignificant associations between leptin and androgen levels are in agreement with previous studies $(42,44-46)$. Further supporting a minor relation between testosterone and leptin, Krotkiewski et al. (48) found no significant changes of leptin levels during anti-androgen treatment in PCOS.

In the present study, we further examined the relations between adiponectin, ghrelin and leptin. Adiponectin levels correlated positively with ghrelin and inversely with leptin in patients, whereas no significant correlations were found in controls. Our findings suggest significantly different regulations of adiponectin, ghrelin and leptin levels in PCOS patients compared with healthy individuals. These findings were supported by significant interactions between patient group and both adiponectin and ghrelin. PCOS patients were characterised by higher levels of testosterone, insulin, HOMA-r and WHR than controls. The correlations between adiponectin, ghrelin and leptin remained significant after adjusting for patient group in multiple regression analyses and adiponectin was shown to be a BMI-independent predictor of ghrelin levels. The inverse relationship between ghrelin and leptin has previously been established in both healthy individuals (49) and PCOS patients (32) and supports findings that the effects of ghrelin on energy homeostasis are opposite to the effects of leptin (49). To our knowledge, only one recent study evaluated the relations between these three peptides in PCOS (32). In agreement with the results of our study, Vrbikova et al. found adiponectin to be an important predictor of ghrelin and vice versa, whereas no significant correlations with leptin levels were found.

In conclusion, obese PCOS patients demonstrated significantly lower adiponectin levels than controls, thus suggesting this subgroup of patients to be at very high risk for the metabolic syndrome. The whole group of PCOS patients as well as subgroups of patients had significantly lower ghrelin levels than weight-matched controls. Furthermore, testosterone was found to have BMI-independent relationships with adiponectin and ghrelin levels. Finally, correlations between adiponectin, ghrelin and leptin differed in PCOS patients and controls, thus suggesting a pathogenic regulation in PCOS.

\section{Acknowledgements}

The study was supported by grants from Ingemann O Buck's Foundation and the Medical Research Foundation in the County of Funen. Allan Flyvbjerg and Jan Frystyk were supported by The Danish Research Council for Health and Disease. The authors thank Bente Tøt, Kirsten Westermann, Donna Arbuckle, Anette Madsen, Rikke Kiilsholm, Hanne Petersen and Joan Hansen for technical assistance.

\section{References}

1 Bjorntorp P. The android woman-a risky condition. Journal of Internal Medicine 1996239 105-110.

2 Revised 2003 consensus on diagnostic criteria and long-term health risks related to polycystic ovary syndrome. Fertility and Sterility 200481 19-25.

3 Douchi T, Ijuin H, Nakamura S, Oki T, Yamamoto S \& Nagata Y. Body fat distribution in women with polycystic ovary syndrome. Obstetrics \& Gynecology 199586 16-519.

4 Kirchengast S \& Huber J. Body composition characteristics and body fat distribution in lean women with polycystic ovary syndrome. Human Reproduction 200116 1255-1260.

5 Ehrmann DA, Barnes RB, Rosenfield RL, Cavaghan MK \& Imperial J. Prevalence of impaired glucose tolerance and diabetes in women with polycystic ovary syndrome. Diabetes Care 1999 22 141-146.

6 Glintborg D, Henriksen JE, Andersen M, Hagen C, Hangaard J, Rasmussen P, Schousboe K \& Hermann AP. The prevalence of endocrine diseases and abnormal glucose tolerance tests in 340 Caucasian, premenopausal women with hirsutism as primary diagnosis. Fertility and Sterility 200482 1570-1579.

7 Fasshauer M \& Paschke R. Regulation of adipocytokines and insulin resistance. Diabetologia 200346 1594-1603.

8 Carmina E, Orio F, Palomba S, Cascella T, Longo RA, Colao AM, Lombardi G \& Lobo RA. Evidence for altered adipocyte function in polycystic ovary syndrome. European Journal of Endocrinology 2005 152 389-394.

9 Diez JJ \& Iglesias P. The role of the novel adipocyte-derived hormone adiponectin in human disease. European Journal of Endocrinology 2003148 293-300.

10 Spranger J, Mohlig M, Wegewitz U, Ristow M, Pfeiffer AF, Schill T, Schlosser HW, Brabant G \& Schofl C. Adiponectin is independently associated with insulin sensitivity in women with polycystic ovary syndrome. Clinical Endocrinology (Oxford) 200461 738-746.

11 Ardawi MS \& Rouzi AA. Plasma adiponectin and insulin resistance in women with polycystic ovary syndrome. Fertility and Sterility $2005831708-1716$.

12 Kondo H, Shimomura I, Matsukawa Y, Kumada M, Takahashi M, Matsuda M, Ouchi N, Kihara S, Kawamoto T, Sumitsuji S, Funahashi T \& Matsuzawa Y. Association of adiponectin mutation with type-2 diabetes: a candidate gene for the insulin resistance syndrome. Diabetes $2002512325-2328$.

13 Otto B, Spranger J, Benoit SC, Clegg DJ \& Tschop MH. The many faces of ghrelin: new perspectives for nutrition research? British Journal of Nutrition 200593 765-771.

14 Tschop M, Weyer C, Tataranni PA, Devanarayan V, Ravussin E \& Heiman ML. Circulating ghrelin levels are decreased in human obesity. Diabetes $200150707-709$. 
15 Ghigo E, Broglio F, Arvat E, Maccario M, Papotti M \& Muccioli G. Ghrelin: more than a natural GH secretagogue and/or an orexigenic factor. Clinical Endocrinology (Oxford) 200562 1-17.

16 Poykko SM, Kellokoski E, Horkko S, Kauma H, Kesaniemi YA \& Ukkola O. Low plasma ghrelin is associated with insulin resistance, hypertension, and the prevalence of type-2 diabetes. Diabetes 2003 52 2546-2553.

17 Broglio F, Gottero C, Benso A, Prodam F, Destefanis S, Gauna C, Maccario M, Deghenghi R, van der Lely AJ \& Ghigo E. Effects of ghrelin on the insulin and glycemic responses to glucose, arginine, or free fatty acids load in humans. Journal of Clinical Endocrinology and Metabolism $2003 \mathbf{8 8} 4268-4272$.

18 Schofl C, Horn R, Schill T, Schlosser HW, Muller MJ \& Brabant G. Circulating ghrelin levels in patients with polycystic ovary syndrome. Journal of Clinical Endocrinology and Metabolism 2002 87 4607-4610.

19 Moran LJ, Noakes M, Clifton PM, Wittert GA, Tomlinson L, Galletly C, Luscombe ND \& Norman RJ. Ghrelin and measures of satiety are altered in polycystic ovary syndrome but not differentially affected by diet composition. Journal of Clinical Endocrinology and Metabolism $2004893337-3344$.

20 Panidis D, Farmakiotis D, Koliakos G, Rousso D, Kourtis A, Katsikis I, Asteriadis C, Karayannis V \& Diamanti-Kandarakis E. Comparative study of plasma ghrelin levels in women with polycystic ovary syndrome, in hyperandrogenic women and in normal controls. Human Reproduction 200520 2127-2132.

21 Pagotto U, Gambineri A, Vicennati V, Heiman ML, Tschop M \& Pasquali R. Plasma ghrelin, obesity, and the polycystic ovary syndrome: correlation with insulin resistance and androgen levels. Journal of Clinical Endocrinology and Metabolism 200287 5625-5629.

22 Sepilian V \& Nagamani M. Adiponectin levels in women with polycystic ovary syndrome and severe insulin resistance. Journal of the Society for Gynecologic Investigationig 200512 129-134.

23 Sieminska L, Marek B, Kos-Kudla B, Niedziolka D, Kajdaniuk D, Nowak M \& Glogowska-Szelag J. Serum adiponectin in women with polycystic ovarian syndrome and its relation to clinical, metabolic and endocrine parameters. Journal of Endocrinological Investigation 200427 528-534.

24 Ferriman D \& Gallwey JD. Clinical assessment of body hair growth in women. Journal of Clinical Endocrinology 196121 1440-1447.

25 Glintborg D, Andersen M, Hagen C \& Hermann AP. Higher bone mineral density in Caucasian, PCOS patients of reproductive age, Positive correlation of testosterone levels with bone mineral density in hirsutism. Clinical Endocrinology (Oxford) 200562 683-691.

26 Frystyk J, Tarnow L, Krarup HT, Parving HH \& Flyvbjerg A. Increased serum adiponectin levels in type 1 diabetic patients with microvascular complications. Diabetologia 200548 1911-1918.

27 Espelund U, Hansen TK, Hojlund K, Beck-Nielsen H, Clausen JT, Hansen BS, Jorgensen JO \& Frystyk J. Fasting unmasks a strong inverse association between ghrelin and cortisol in serum: studies in obese and normal-weight subjects. Journal of Clinical Endocrinology and Metabolism 200590 741-746.

28 Lykkesfeldt G, Bennett P, Lykkesfeldt AE, Micic S, Moller S \& Svenstrup B. Abnormal androgen and oestrogen metabolism in men with steroid sulphatase deficiency and recessive X-linked ichthyosis. Clinical Endocrinology (Oxford) 198523 385-393.

29 Bland JM \& Altman DG. Transformations, means, and confidence intervals. British Medical Journal 19963121079.

30 Panidis D, Kourtis A, Farmakiotis D, Mouslech T, Rousso D \& Koliakos G. Serum adiponectin levels in women with polycystic ovary syndrome. Human Reproduction 200318 1790-1796.

31 Orio F, Jr, Palomba S, Cascella T, Milan G, Mioni R, Pagano C, Zullo F, Colao A, Lombardi G \& Vettor R. Adiponectin levels in women with polycystic ovary syndrome. Journal of Clinical Endocrinology and Metabolism 200388 2619-2623.

32 Vrbikova J, Dvorakova K, Hill M, Vcelak J, Stanicka S, Vankova M, Sramkova D, Vondra K, Bendlova B \& Starka L. Determinants of Circulating Adiponectin in Women with Polycystic Ovary Syndrome. Gynecologic and Obstetric Investigation 200560 155-161.

33 Scherer PE, Williams S, Fogliano M, Baldini G \& Lodish HF. A novel serum protein similar to C1q, produced exclusively in adipocytes. Journal of Biological Chemistry 1995270 26746-26749.

34 Nishizawa H, Shimomura I, Kishida K, Maeda N, Kuriyama H, Nagaretani H, Matsuda M, Kondo H, Furuyama N, Kihara S, Nakamura T, Tochino Y, Funahashi T \& Matsuzawa Y. Androgens decrease plasma adiponectin, an insulin-sensitizing adipocytederived protein. Diabetes $2002512734-2741$.

35 Bottner A, Kratzsch J, Muller G, Kapellen TM, Bluher S, Keller E, Bluher M \& Kiess W. Gender differences of adiponectin levels develop during the progression of puberty and are related to serum androgen levels. Journal of Clinical Endocrinology and Metabolism 200489 4053-4061.

36 Page ST, Herbst KL, Amory JK, Coviello AD, Anawalt BD \& Matsumoto AM. Testosterone administration suppresses adiponectin levels in men. Journal of Andrology 200526 85-92.

37 Broglio F, Gottero C, Arvat E \& Ghigo E. Endocrine and nonendocrine actions of ghrelin. Hormone Research $2003 \mathbf{5 9}$ 109-117.

38 Orio F, Jr, Lucidi P, Palomba S, Tauchmanova L, Cascella T, Russo T, Zullo F, Colao A, Lombardi G \& De Feo P. Circulating ghrelin concentrations in the polycystic ovary syndrome. Journal of Clinical Endocrinology and Metabolism $2003 \mathbf{8 8} 942-945$.

39 Legro RS. Diagnostic criteria in polycystic ovary syndrome. Seminars in Reproductive Medicine 200321 267-275.

40 Barreiro ML \& Tena-Sempere M. Ghrelin and reproduction: a novel signal linking energy status and fertility? Molecular and Cellular Endocrinology 2004226 1-9.

41 Gambineri A, Pagotto U, Tschop M, Vicennati V, Manicardi E, Carcello A, Cacciari M, De Iasio R \& Pasquali R. Anti-androgen treatment increases circulating ghrelin levels in obese women with polycystic ovary syndrome. Journal of Endocrinological Investigation 200326 629-634.

42 Carmina E, Ferin M, Gonzalez F \& Lobo RA. Evidence that insulin and androgens may participate in the regulation of serum leptin levels in women. Fertility and Sterility 199972 926-931.

43 Remsberg KE, Talbott EO, Zborowski JV, Evans RW \& McHughPemu K. Evidence for competing effects of body mass, hyperinsulinemia, insulin resistance, and androgens on leptin levels among lean, overweight, and obese women with polycystic ovary syndrome. Fertility and Sterility $2002 \mathbf{7 8} 479-486$.

44 Mantzoros CS, Dunaif A \& Flier JS. Leptin concentrations in the polycystic ovary syndrome. Journal of Clinical Endocrinology and Metabolism $1997 \mathbf{8 2} 1687-1691$.

45 Rouru J, Anttila L, Koskinen P, Penttila TA, Irjala K, Huupponen R $\&$ Koulo M. Serum leptin concentrations in women with polycystic ovary syndrome. Journal of Clinical Endocrinology and Metabolism 199782 1697-1700.

46 Saleh HA, El-Nwaem MA, El-Bordiny MM, Maqlad HM, El-Mohandes AA \& Eldaqaq EM. Serum leptin elevation in obese women with PCOs: a continuing controversy. Journal of Assisted Reproduction and Genetics 200421 361-366.

47 Telli MH, Yildirim M \& Noyan V. Serum leptin levels in patients with polycystic ovary syndrome. Fertility and Sterility 200277 932-935.

48 Krotkiewski M, Landin K, Dahlgren E, Janson PO \& Holm G. Effect of two modes of anti-androgen treatment on insulin sensitivity and serum leptin in women with PCOS. Gynecologic and Obstetric Investigation $2003 \mathbf{5 5} 88-95$.

49 Yildiz BO, Suchard MA, Wong ML, McCann SM \& Licinio J. Alterations in the dynamics of circulating ghrelin, adiponectin, and leptin in human obesity. Proceedings of the National Academy of Sciences USA $200410110434-10439$.

Received 14 March 2006

Accepted 8 May 2006 